\title{
Competences representing the goal of foreign language learning: is the approach real?
}

\author{
[Les competences en objectif d'apprentissage des langues : \\ mythe ou realite?]
}

\author{
Svetlana Kashchuk
}

DOI: 10.18355/XL.2019.12.01XL.04

\begin{abstract}
The article aims to question the modern state of theoretical basics of foreign language teaching. The author sufficiently grounds the importance of foreign language learning goal precision in the very introduction as this methodological category normally defines approaches, means and content of learning, altogether bringing (or not) the learner to the wanted result. Within the span of the article, the evolution of linguadidactical methods addressing the needs of a constantly dynamic society, is examined. The author traces the transformation of the goal of foreign language learning, from logical thinking improvement (grammar and translation method) to the formation of general and language-specific competences (competence approach). Upon the analysis of the transformation process and consecutive methods in use, the means and the content of foreign language learning, and in compliance with modern linguadidactic specialists' opinions, the author concludes that the notion of competence is artificial in its essence. This ousts it from being the ground of effective foreign language learning process. As a solution to the poor efficiency choice of setas-a-goal-competence problem, the author suggests alternative viewpoint of a Russian scholar Yefim Passov, who claimed that the goal of education is a man as such, not the competence formation process. Thus it's not just communication using a number of artificially brought about competences, but the human being Homo moralis, a bearer of foreign language culture and capable of intercultural dialoguing, should be set as a goal.
\end{abstract}

Key words: foreign language learning goals, communicative competence, modern methods of foreign language teaching, linguadidactics methods evolution.

\section{Résumé}

L'importance de l'article repose sur la nécessité d'étudier la situation actuelle dans la didactique des langues. Dans l'introduction, l'auteur prouve l'importance de la définition de l'objectif d'enseignement des langues parce que c'est cette catégorie didactique qui définit les méthodes, les outils d'apprentissage, le contenu d'enseignement et surtout les résultats à attendre. Dans son développement, la didactique des langues a connu plusieurs étapes dont chaque nouvelle s'opposait aux précédentes : en commençant par la méthode grammaire-traduction, passant par la méthodologie naturelle (ou directe), l'approche SGAV (structuro-global-audiovisuelle), l'approche communicative, et en arrivant à l'approche par compétences dans une démarche actionnelle. Dans toutes ces étapes, l'objectif d'apprentissage des langues étrangères se transformait pour répondre aux besoins de la société, elle-même en changement et en mutation permanente. En étudiant les points de vue des spécialistes en didactique des langues, l'auteur fait la conclusion, que dès son apparition, la notion de la «compétence »s'est avérée artificielle. C'est pourquoi il est difficile de construire un système éducatif efficace et fiable qui repose sur les compétences, elles-mêmes difficiles à identifier. Comme solution, nous proposons le point de vue d'un savant russe Efim Passov qui insiste sur l'idée que l'objectif de 
l'apprentissage c'est l'Homme (Homo moralis) doté d'une culture langagière étrangère et capable de mener un dialogue interculturel et pas du tout le processus de la formation des compétences.

Mots-clés : objectif d'enseignement, compétence de communication, actuelle didactique des langues, évolution de la didactique des langues

\section{Introduction}

On prête autant d'attention à l'objectif d'apprentissage parce que tout ce qu'on fait sans but n'a pas de sens. Faire quoique ce soit sans résultat final prévu, c'est perdre du temps. Et c'est surtout dangereux dans le domaine de l'éducation où il faut désigner bien à l'avance le résultat désiré et les moyens pour l'atteindre. En effet, le changement des objectifs d'apprentissage des langues provoque inévitablement le changement des méthodes, des outils d'apprentissage, du contenu d'enseignement et surtout des résultats. Donc, nul ne peut nier l'importance de la précision des objectifs d'apprentissage en général et des langues en particulier.

A travers les siècles, les philosophes, pédagogues, linguistes et didacticiens essayaient de définir l'objectif d'apprentissage des langues ainsi que les moyens pour l'atteindre. Mais cette tâche n'a jamais été complètement accomplie et reste aujourd'hui la préoccupation majeure des spécialistes de l'éducation. Parcourons l'évolution et la transformation des objectifs d'apprentissage des langues au fil du temps, ce qui nous permettra de voir plus clair où on est arrivé au XXIe siècle.

Dans le «Dictionnaire de didactique du français langue étrangère et seconde », l'objectif de l'apprentissage des langues est défini de la manière suivante : «En didactique des langues, l'objectif est le résultat idéal de l'enseignement et de l'apprentissage. La notion d'objectif est très liée aux moyens mis en place pour sa réalisation: stratégies, types de méthodes, contenus d'enseignement, activités pédagogiques, progression, système d'évaluation etc. On distingue en général deux types d'objectifs : les objectifs globaux et les sous-objectifs séquentiels, comme des tâches à accomplir pour réaliser à la fin l'objectif global (Cuq, 2003: 180). » Donc, il ne nous reste qu'à définir ce « résultat idéal».

D'après la tradition éducative européenne, l'enseignement du latin dans toutes les universités a été obligatoire pendant plus de mille ans, ce qui a défini l'apprentissage d'autres langues. L'apparition du grec à la Renaissance, n'a rien changé. L'idée du développement de la langue vivante était complètement exclue, la grammaire des langues européennes était soumise à la grammaire du latin. En conséquence, pendant les trois derniers siècles, jusqu'au début du XIXe siècle, en Europe, aussi qu'en Russie, l'enseignement du latin et du grec dans des établissements scolaires et universitaires dominaient l'enseignement d'autres langues européennes à tel point, qu'on enseignait les langues étrangères vivantes d'après le modèle d'enseignement du latin et du grec sans tenir compte de la différence et de la fonction de ces langues. L'enseignement se basait généralement sur la méthode grammaire-traduction, qui avait une place privilégiée non seulement dans l'enseignement des langues anciennes mais aussi dans l'enseignement des langues étrangères vivantes. On souligne que «pendant longtemps l'étude d'une langue vivante dans un lycée ou un collège n'a été que le complément de la culture humaniste et classicisante proposée à l'élève : le but de l'enseignement de l'anglais, de l'allemand, de l'espagnol ou de l'italien était de permettre à l'élève de lire, ou tout au moins de savoir traduire Shakespeare, Goethe, Cervantes et Dante. Accessoirement l'élève pouvait en profiter pour apprendre à parler la langue, mais on ne le lui demandait pas expressément et on ne faisait rien pour l'y aider (Reboullet, 1971). » A cette époque-là, le contenu

XLinguae, Volume 12 Issue 1XL, January 2019, ISSN 1337-8384, eISSN 2453-711X 
d'enseignement ne comportait que des règles de grammaire et des exercices de traduction. Et, comme le montrent bien Tardif Jacques et Dubois Bruno, « ... on estimait alors que ces apprentissages permettaient de développer une logique formelle, une pensée structurée et nuancée, un raisonnement argumentatif cohérent ... (Dubois, 2013 ;Tardif, 2013). » C'est-à-dire, le développement d'une logique formelle était l'objectif d'enseignement des langues étrangères jusqu'au début du XIXe siècle.

$\mathrm{Au}$ XIXe siècle, en didactique des langues, on voit apparaître la méthode naturelle (ou directe) d'après laquelle l'apprenant devait acquérir une langue étrangère directement, d'une façon dont les enfants apprennent leur langue maternelle. A cette période-là, on croyait qu'il n'y avait pas de moyens spécifiques pour apprendre une langue. C'est en exerçant son activité de producteur de langage que l'élève apprend la langue (Lèmery, 1993). Les représentants de cette méthode soulignaient qu'il fallait reconstruire autours de l'apprenant la situation imitant celle de la satiation naturelle de l'acquisition de la langue maternelle par l'enfant. D'où son nom «méthode naturelle ». Les partisans de la méthode naturelle ne prêtaient pas beaucoup d'attention ni à l'enseignement de l'écrit, ni, non plus, à l'enseignement de la lecture. Ils croyaient qu'après avoir appris à communiquer oralement en langue étrangère, les apprenants pourront eux-mêmes acquérir facilement l'écrit et la lecture, ce qui était, évidemment, faux. La langue maternelle a été complètement exclue du processus d'apprentissage. L'acquisition se faisait par l'expérience à même la vie et les milieux. En Russie cette méthode est aussi connue sous le nom de « la méthode de la gouvernante. » ${ }^{1}$

En ce qui concerne l'objectif principal de l'apprentissage d'une langue étrangère, il a bien changé par rapport à la méthode grammaire-traduction. Maintenant, on apprenait une langue étrangère à l'école parce que c'était «le déploiement des procédés et réseaux de communication (la correspondance, le journal de classe, le texte libre, l'enquête, l'exposé, le débat...) qui induisent le processus de l'apprentissage (Lèmery, 1993). » Désormais, les apprenants devaient pouvoir utiliser la langue étrangère comme un outil de communication (dans une situation d'apprentissage) et pas forcément comme le développement d'une logique formelle.

Dans les années $60 \mathrm{du}$ siècle dernier, est apparue la méthode audiovisuelle (MAV) qui, certainement, a été un pas en avance par rapport aux méthodes précédentes. On nomme cette problématique méthodologique d'enseignement-apprentissage des langues SGAV (structuro-global-audiovisuelle): la langue étrangère est présentée sous forme de structures, l'apprentissage est construit autour de l'utilisation du son et de l'image. MAV se base sur la théorie psychologique béhavioriste et la linguistique structurée. L'élève apprend les structures linguistiques en les répétant plusieurs fois dans des situations éducatives différentes. L'apprentissage s'effectue d'après le schéma «stimule - réaction». La priorité est donnée à la communication orale en interaction en associant le verbal et le paraverbal. Les images et le son mettaient les apprenants dans la situation langagière proche de la réelle. Contrairement à la méthode grammaire-traduction, la langue a été considérée comme une matière vivante dans toute son intégralité.

\footnotetext{
${ }^{1}$ Dans les familles aristocrates russes durant des siècles on était accoutumé à parler français. Pour cette raison, on employait souvent les jeunes françaises natives comme nounou, pour que les enfants apprennent la langue française dès le plus jeune âge. Ces femmes, le plus souvent ne connaissant pas le russe, s'occupaient des enfants en leur parlant français. En russe on les appelait «гувернантка» ("gouvernante"). De nos jours, nous assistons à la réapparition de cette pratique dans les familles russes aisées.
} 
Cette méthodologie d'enseignement des langues a très vite dominé la France et a influencé l'enseignement du FLE (français langue étrangère). Les Français appellent aussi cette méthode VIF, comme le premier manuel d'apprentissage du français langue étrangère basé sur MAV «Voix et image de France». On croyait avoir trouvé la méthode révolutionnaire et idéale pour pouvoir enseigner à succès les langues étrangères.

Les attentes étaient fortes, mais MAV a mené à un échec car l'objectif d'apprentissage d'une langue étrangère - la communication libre orale - n'a pas été atteint. Le schéma "stimule - réaction » qui s'est très bien montré dans la situation d'apprentissage, n'a pas pu fonctionner dans la vie réelle. Exemple : si dans la situation d'apprentissage en classe à la question «Comment ça va ? » l'apprenant donnait la réponse «Merci, ça va bien », dans la vie de tous les jours, à la question « Alors, ça roule ? » il ne trouvait pas de réponse. D'après J.-P. Cuq, «le transfert de l'acquis espéré de la salle de classe au monde extérieur était loin de s'opérer » (Cuq, 2003: 28). En ce qui concerne la notion de communication, elle, non plus, n'a pas été précisée.

La méthode audiovisuelle très répandue au XXe siècle mais très vite critiquée a cédé sa place au début du XXIe siècle à la méthode communicative où la communication en tant que processus de transmission de l'information en langue étrangère a été encore plus accentuée. On précise l'objectif d'apprentissage d'une langue et on fait apparaître la compétence communicative. C'est en cette période de méthode communicative, qu'on rencontre pour la première fois dans le domaine de la didactique des langues la notion de « compétence » issue du monde du travail où elle est considérée comme « une capacité d'affronter l'inédit et le changement permanent, de faire face à l'incertitude » (Le Boterf, 2000: 23). Dans le dictionnaire multimédia « Larousse » la notion de « compétence » est présentée de la manière suivante: «Compétence: nom féminin (bas latin competentia, juste rapport) capacité reconnue en telle ou telle matière en raison de connaissances possédées et qui donne le droit d'en juger (Larousse, 2018). » Donc, dans le monde du développement rapide des technologies et changement permanent des situations sociales et politiques, il ne suffit plus de posséder des connaissances, il faut savoir gérer les situations imprévues et même pouvoir les prévoir. Et c'est ce terme "compétence », qui s'est transféré du monde de travail au monde de la didactique des langues. Désormais, on parle de la compétence communicative.

La notion de la «compétence communicative» a été introduite dans la didactique des langues par Dell Hymes. D'après lui, la compétence communicative doit comporter en plus de la composante linguistique les savoirs socioculturels (c'està-dire, il fallait enseigner aussi les règles d'utilisation de la langue dans des situations de communication). Plus tard, cette notion de compétence communicative a été précisée plusieurs fois. E. Berard note dans son livre «L'approche communicative. Théorie et pratiques » que tous les didacticiens décrivaient la compétence de communication à sa façon et dégageaient le nombre tout à fait différent de ses composants (Berard E., 1991). E. Berard propose de comparer les précisions de la notion de «compétence communicative» données par D. Hymes (Hymes, 1984), C. Swain (Swain, 1980) et S. Moirand (Moirand, 1982). 


\section{Compétence communicative (CC)}

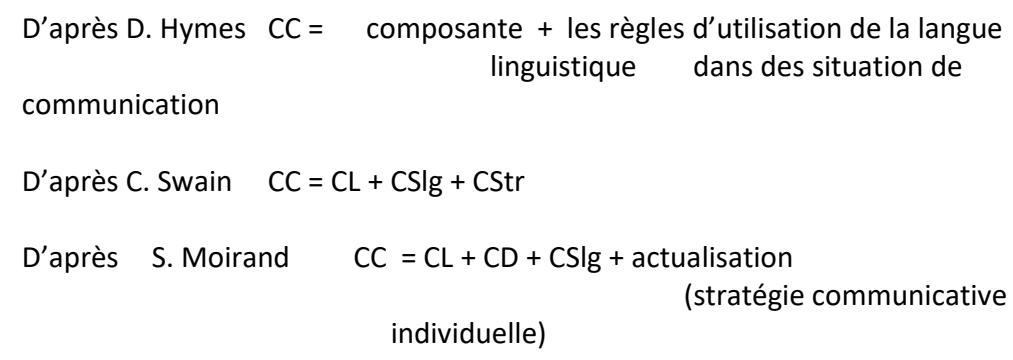

\footnotetext{
$\mathrm{CC}$ - compétence communicative;

$\mathrm{CL}$ - compétence linguistique;

CSlg - compétence sociolinguistique;

CStr - compétence stratégique;

$\mathrm{CD}$ - compétence discursive.
}

\section{Image 1. L'analyse comparative du nombre des composantes de la notion de « compétence communicative»}

Comme nous le voyons bien, à l'époque de la domination de l'approche communicative dans l'enseignement des langues, l'objectif d'apprentissage des langues n'a pas du tout été clair, l'objectif en «compétence communicative » était très flou et la question «Qu'est-ce qu'apprendre une langue étrangère ? " était toujours sans réponse.

Dans le nouveau contexte politique, les changements de l'Europe qui est devenue l'Union européenne avec l'espace éducatif commun dont la Russie fait désormais partie, ont provoqué les changements dans la didactique des langues. Aujourd'hui, on parle beaucoup du multilinguisme et on en a vraiment besoin. On souligne une demande en faveur de la société européenne multilingue contemporaine de citoyens capables d'utiliser les langues européennes pour réussir leur vie professionnelle et privée (Kozarenko, 2017). E. Rosen remarque, qu'on prépare les apprenants en classe à pouvoir s'intégrer dans les pays d'Europe qu'ils seront amenés à fréquenter pour une durée assez longue (pour effectuer une partie de leurs études à l'étranger, pour y effectuer une partie de leur carrière, etc.) : on ne se contente plus de former un "étranger de passage » capable de communiquer dans des situations attendues, on souhaite aider un apprenant à devenir un utilisateur de la langue, un acteur social à même de s'intégrer efficacement dans un autre pays (Rosen, 2006).

Donc, la question de l'apprentissage des langues et surtout de la réussite dans ce domaine est devenue plus que jamais importante. Un état des lieux en la matière était très urgent au début du XXIe siècle. Il fallait bien préciser les objectifs et les évaluations pour former le "nouvel étranger » qui est "l'étudiant européen voyageur» (Murphy-Lejeune, 2003). 
Une tentative a été entreprise par une équipe du CECRL (Cadre Européen Commun de référence pour les langues). Mais au troisième millénaire, après une dizaine d'années de recherche menées sous la direction du Conseil de l'Europe dans le domaine de la didactique des langues, l'objectif d'apprentissage des langues en Union européenne est resté aussi flou qu'avant : «le but de l'apprentissage d'une langue est de faire de l'apprenant un utilisateur compétent et expérimenté (CECRL, 2001). » Il est clair, que cet énoncé est fort applicable à toutes les périodes précédentes d'existence de la didactique des langues.

La demande de la société européenne nouvelle de former l'étudiant européen voyageur et pour qu'il devienne après le nouvel étranger a exigé de préciser l'ensemble des résultats à atteindre dans le domaine de l'apprentissage - enseignement des langues étrangères. Pour cela, on a développé la notion de la « compétence en langue » en décrivant en détails ses composantes : savoir-faire ;

-les compétences générales: savoir, aptitude et savoir-faire, savoir-être,

-les compétences communicatives langagières : compétences linguistiques, compétences sociolinguistiques, compétences pragmatiques (CECRL, 2001).

Il y a une vingtaine d'années que la didactique des langues se base sur l'approche par compétences souvent désignée sous le sigle APC. Désormais, dans les établissements éducatifs, les programmes d'étude en général et des langues en particulier sont rédigés en termes de compétences. Tous les manuels des langues sont fortement attachés aux compétences. Et cette pratique commence à être de plus en plus critiquée sur tout l'espace éducatif européen commun, parce que souvent on fait porter l'évaluation des acquis langagiers sur «ce qu'est l'élève » plutôt que sur «ce qu'il a appris », sur ce qu'il a appris « en dehors de l'école » plutôt que sur ce qu'il a appris «à l'école» (De Ketele, 2013).

A notre avis, cette situation est provoquée par la non-précision de l'objectif d'enseignement-apprentissage et surtout de la non-précision de la notion de « compétence en langues », celle-ci souvent considérée tout simplement comme «le développement d'un savoir agir » (Dubois, 2013 ; Tardif, 2013).

Un pédagogue et philosophe russe Ephim Izlievitch Passov insiste sur l'idée que nous avons aujourd'hui l'objectif d'enseignement-apprentissage directif et non réel en «compétences - fantômes ». Il souligne qu'aujourd'hui les compétences représentent pour l'enseignement la même chose que le gaz phlogistique ${ }^{2}$ pour l'alchimie durant presque 150 ans (Passov, 2011). Les savants des siècles derniers n'arrivaient pas à décrire ce gaz car il n'a jamais existé en réalité. Il est fort possible, que les didacticiens d'aujourd'hui n'arrive pas à décrire les compétences comme objectif d'apprentissage des langues parce qu'elles n'existent pas non plus? C'est peut-être aussi pour cette raison que les objectifs d'enseignement des langues n'ont jamais été atteints durant toute la longue histoire de la didactique des langues.

\section{Quelle est la solution ?}

Premièrement, exclure les compétences comme objectif-fantôme d'enseignement-apprentissage des programmes et manuels scolaires et universitaires. Cela permettra de rendre le système d'apprentissage des langues moins formel et, comme conséquence, beaucoup plus efficace.

L'objectif a du sens s'il a son objet d'application. En éducation c'est l'apprenant, c'est l'être humain. L'objectif est toujours interprété à travers le résultat à atteindre. Souvent, dans le résultat on remplace l'objectif essentiel (dans notre cas

${ }^{2}$ Fluide imaginaire provoquant la combustion, pour les anciens alchimistes. XLinguae, Volume 12 Issue 1XL, January 2019, ISSN 1337-8384, eISSN 2453-711X 
c'est l'Homme) par les tâches pragmatiques - formations des savoirs et des savoirfaire en langue étrangère. Il est à souligner que ces tâches, certainement importantes, ne sont que des instruments pour atteindre l'objectif essentiel. Alors, pour préciser l'objectif d'apprentissage des langues il faut s'éloigner du pragmatisme et s'approcher de l'humanisme, parce qu'il est impossible de décrire le processus humain (qui est l'enseignement des langues en particulier et l'éducation en général) en terme pragmatique.

Il est temps aujourd'hui de passer de l'enseignement des langues à l'éducation langagière et, en conséquence de changer d'objectif d'apprentissage: passer de la communication en langues étrangères d'un apprenant au dialogue interculturel de l'Homo moralis - l'étudiant européen voyageur.
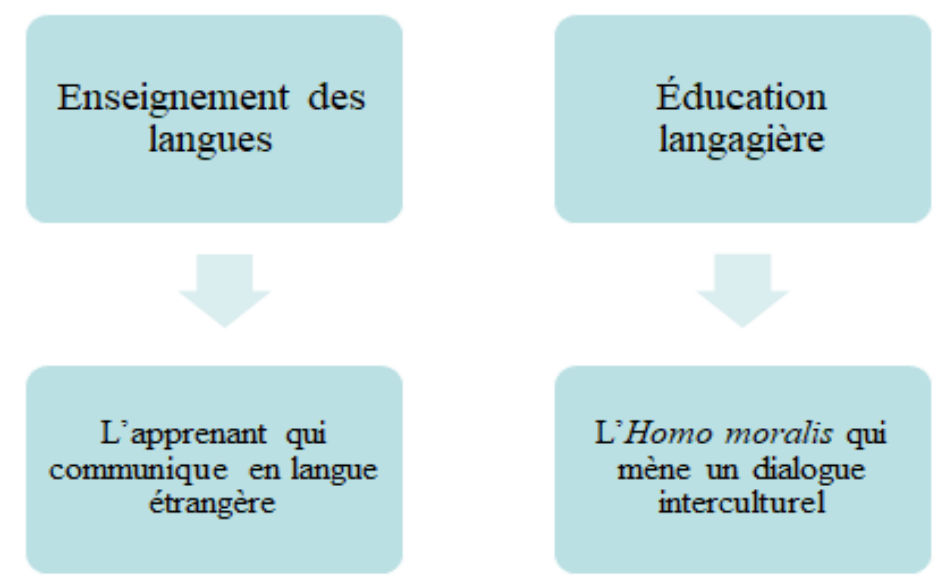

\section{Image 2. Le schéma du passage de l'enseignement des langues à l'éducation langagière}

Ephim Izlievitch Passov insiste sur l'idée que l'objectif général de l'enseignement des langues c'est l'Homo moralis, c'est-à-dire l'apprenant qui a acquis la culture étrangère langagière comme contenu de l'apprentissage. Si on regarde l'Encyclopédie philosophique (1983), le contenu y est considéré comme "état intérieur, ensemble de processus ». D'après Passov E. I., le contenu dans l'apprentissage des langues - la culture étrangère langagière - représente quatre processus: cognitif, développant, éducateur, d'étude.

le processus cognitif: on apprend à utiliser la culture étrangère langagière dans le dialogue avec sa culture langagière native (objet de l'acquisition aspect culturel: les faits de la culture étrangère, y compris la langue comme une partie intégrante de la culture étrangère toujours en dialogue avec sa propre culture);

le processus développant: on apprend à apprendre, à s'intéresser à la langue et la culture étrangères (objet du développement - aspect psychologique : capacité de mémoriser, imaginer, prévoir, remplir les lacunes, dialoguer, construire une expérience positive de l'utilisation de la langue étrangère) ; 
le processus éducateur : on apprend à appliquer dans le dialogue interculturel le potentiel moral et culturel acquis pendant les études (objet du développement - aspect pédagogique : la culture esthétique, la culture éthique, la culture écologique, la morale, la foi, la tolérance, le patriotisme et l'internationalisme etc.) ;

le processus d'étude : on apprend à mener le dialogue interculturel en utilisant les activités langagières productives telles que l'écrit et l'oral (le monologue/le dialogue) aussi que les activités langagières réceptives telles que la lecture et l'écoute (objet de l'acquisition - aspect social : savoir lire, écrire, écouter, parler).

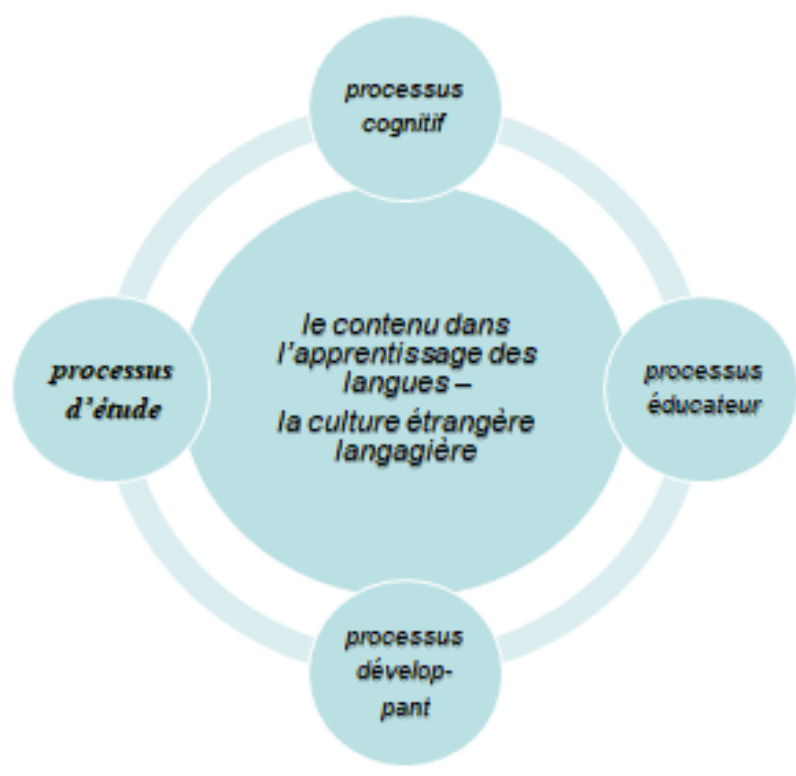

Image 3. Le schéma du contenu dans l'apprentissage des langues pour former la culture étrangère langagière des apprenants en langues étrangères

Quand tous ces quatre processus s'intègrent, ils créent le champ de l'éducation langagière efficace dont l'objectif est l'Homo moralis qui est aussi l'étudiant européen voyageur capable de mener un dialogue interculturel pour devenir le nouvel étranger.

\section{Conclusion}

Nous avons parcouru brièvement l'histoire du développement de la didactique des langues et analysé le changement des objectifs d'apprentissage des langues selon la demande de la société. Cet article reste une invitation aux réflexions et à la discussion au sujet d'objectif d'apprentissage des langues au XXIème siècle, le siècle de la communication rapide et sans frontière (grâce au développement des technologies numériques), le siècle où on a besoin plus que jamais de comprendre l'étranger (dans la vie réel ou sur la Toile) et de pouvoir mener le dialogue XLinguae, Volume 12 Issue 1XL, January 2019, ISSN 1337-8384, eISSN 2453-711X 
interculturel. L'organisation du processus éducatif intégrant le processus cognitif, le processus développant, le processus éducateur et le processus d'étude permettra d'atteindre ce nouvel objectif d'apprentissage et notamment, permettra à l'apprenant de :

- remarquer chez l'étranger pas seulement les points différents, mais aussi les points communs;

- analyser les évènements, les gens et leurs les comportements pas seulement de son propre point de vue, mais du point de vue de la culture étrangère;

- changer ses points de vue au fur et à mesure de l'acquisition de la culture étrangère, ne pas utiliser les stéréotypes ;

- remarquer le nouveau dans les choses habituelles ;

- interpréter et comprendre les valeurs de l'autrui ;

- mieux comprendre sa propre culture à travers la compréhension de la culture étrangère ; étrangère.

- apprendre la langue étrangère comme une partie intégrante de la culture

\section{Bibliographic references}

BERARD, E. 1991. L'approche communicative. Théorie et pratiques. Paris : CLE International. ISBN-10: 2190333520.

Cadre Européen Commun de référence pour les langues: apprendre, enseigner, évaluer. 2001. Strasbourg: Division des Politiques Linguistiques. Available online: https://rm.coe.int/168069782b .

CANALE, M. - SWAIN, M. 1980. Theoretical bases of communicative approaches to second language teaching and testing. In: Applied Lingusitics. pp. 1-47.

CUQ, J.-P. 2003. Dictionnaire de didactique du français langue étrangère et seconde. Paris : CLE International. ISBN : 209-033972-1.

DE KETELE, J.-M. 2013. Présentation. Evaluation et enseignement : pour quels objectifs ? par quels moyens? Dans: Revue française de linguistique appliquée 2013/1 (Vol. XVIII), pp. 3-8. ISSN 1875-368X.

DUBOIS, B. - TARDIF, J. 2013. De la nature des compétences transversales jusqu'à leur évaluation : une course à obstacles, souvent infranchissables. Dans : Revue française de linguistique appliquée, 2013/1 (Vol. XVIII). pp. 29-45. Available online: https://www.cairn.info/revue-francaise-de-linguistique-appliquee-2013-1-page-29.htm Encyclopédie Larousse. 2018. Available online: http://www.larousse.fr/ .

Encyclopédie philosophique. 1983. Sous la direction de Il ichyov L.F., Fedoseev P.N., Kovalyov S.M., Panov V.G. - Moscou: Sovetskaya e`ncziklopediya.

HYMES, D. 1984. On communicative competence. In : Sociolinguistics. Texte traduit dans «Vers la compétence de communication». Paris : Hatier. pp. 17-118. ISSN 0710-0167.

KOZARENKO, O. 2017. Évaluer les compétences de recherche scientifique en langue française. Dans: Revue japonaise de didactique du français. № Numéro spécial. pp. 506-1-506-14. Available online: http://sjdf.org/pdf/cap2017kyoto_actes.pdf.

LE BOTERF, G. 2000. Compétence et navigation professionnelle. Paris : Edition d'organisation. ISBN : 2-7081-2445-5.

LEMERY, J. 1993. Méthode naturelle pour l'apprentissage des langues. - Le Nouvel Educateur $\mathrm{n}^{\circ} 53$, novembre 93. Available online: https://www.icemfreinet.fr/archives/ne/ne/53/dossier-53.pdf .

MOIRAND, S. 1982. Enseigner à communiquer en langue étrangère. Paris: Hachette. ISBN 978-2-01-008787-5.

MURPHY-LEJEUNE, E. 2003. L'Etudiant européen voyageur : Un nouvel étranger. - Paris : Didier. ISBN-10: 2278052705. 
PASSOV, E. I. 2011. Le gaz phlogistique dans l'enseignement ou les compétences en objectif d'apprentissage. - Elecz : MUP "Tipografiya" g.El'cza. ББК 81.47.43P30.

REBOULLET, A. 1971. Guide pédagogique pour le professeur de français langue étrangère. Sous la direction d'André Reboullet. Paris : Hachette. ISBN : 2-01-0004564.

ROSEN, E. 2006. Le point sur le Cadre européen commun de référence pour les langues. Paris : CLE Internationale. ISBN : 978-2-09-03334-3.

Words: 4242

Characters: 29120 (16,2 standard pages)

Prof. Dr. Svetlana Kashchuk, PhD.

Head of Foreign Languages Department

Associate Professor

School of Public Administration,

Lomonosov Moscow State University

1-52, Leninskiye Gory, Moscow, GSP-1

119991

Russia

kashchuk@spa.msu.ru 